\title{
Investigating the zinc ion adsorption capacity of a chitosan/ $\beta$-cyclodextrin complex in wastewater
}

\author{
Jiaxin Liu ${ }^{1}$, Siqi Wang ${ }^{1}$, Xiuqing Ding ${ }^{1}$, Jingyi $\mathrm{Fu}^{1}$, and Jun Zhao ${ }^{1}$ \\ ${ }^{1}$ College of Food Science and Engineering,Changchun University,130022, Changchun,China
}

\begin{abstract}
To decrease the amount of $\mathrm{Zn}^{2+}$ in industrial waste water, in this study, $\beta$-cyclodextrin ( $\beta$-CD) was first modified and then used to obtain a $\beta$-cyclodextrin polymer $(\beta-\mathrm{CDP})$. The effects of reaction temperature and reaction time of $\beta$-CD with citric acid (CA), polyethylene glycol 400 (PEG-400), and disodium hydrogen phosphate $\left(\mathrm{NaH}_{2} \mathrm{PO}_{4}\right)$ on the amount of $\beta$-CDP produced were investigated. The results showed that at a reaction temperature of $145^{\circ} \mathrm{C}$ and a reaction time of $4.5 \mathrm{~h}, 6.58 \mathrm{~g}$ of $\beta$-CDP was produced. Then, chitosan (CTS) was crosslinked with $\beta$-CDP using glutaraldehyde to prepare a chitosan $/ \beta$-cyclodextrin $(\mathrm{CTS} / \beta-\mathrm{CDP}$ ) complex. The mass ratio of CTS to $\beta$-CDP, reaction temperature, reaction time, and amount of added glutaraldehyde were used as the main variables to examine the $\mathrm{Zn}^{2+}$ adsorption rate and adsorption capacity of the composites prepared in this study. The optimum experimental conditions were as follows: a mass ratio of $3: 10$, a reaction temperature of $80^{\circ} \mathrm{C}$, a reaction time of $90 \mathrm{~min}$, and $2 \mathrm{~mL}$ of glutaraldehyde. Under these optimal conditions, the adsorption amount and adsorption rates of $\mathrm{Zn}^{2+}$ using CTS/ $\beta$-CDP complex were respectively $97.70 \mathrm{mg} \cdot \mathrm{g}^{-1}$ and $78.92 \%$.
\end{abstract}

\section{Introduction}

The mechanical manufacturing, papermaking, chemical, and battery manufacturing industries produce large amounts of heavy metal ions such as $\mathrm{Zn}^{2+}$, which is a highly toxic, non-degradable, and persistent pollutant. It causes serious environmental damage and can also enter humans through the food chain when it pollutes rivers and lakes, which seriously endangers human health ${ }^{[1-2]}$. Currently, common methods for removing $\mathrm{Zn}^{2+}$ from waste water include electrolysis, ion exchange, chemical precipitation, adsorption, and electrodialysis ${ }^{[3-4]}$. However, these methods are complicated and produce large amounts of solid waste, and are plagued by high costs and energy consumption. CTS and $\beta-C D$ are natural products with high adsorption abilities that do not cause secondary water pollution. Thus, in recent years, they have been used to treat domestic and industrial wastewater containing heavy metal ions, dyes, phenols, organic matter, and similar pollutants. CTS is abundant in nature, and is the only natural alkaline polysaccharide ${ }^{[5-7]}$. Its molecular chain contains many functional groups, including amino and hydroxyl groups, and it can electrostatically adsorb or complex with heavy metal ions. When combined with heavy metals in water ${ }^{[8]}$, it has an excellent adsorption ability and a high mechanical strength, but it does not have a selective adsorption. The structure of $\beta-\mathrm{CD}$ contains a hydrophobic cavity which, along with its peripheral hydroxyl groups, can remove heavy metal ions from aqueous systems ${ }^{[9-12]}$. Modified $\beta$-CDP maintains the cavity and recognition ability of
$\beta-\mathrm{CD}$, and also has good mechanical strength and stability [13], but it is difficult to recover due to its high water solubility ${ }^{[14]}$. Therefore, in this paper, $\beta$-CDP was cross-linked with the CTS molecular chain to obtain a cyclodextrin-modified CTS derivative to obtain a CTS/ $\beta$-CDP complex with both desirable properties to reduce the content of $\mathrm{Zn}^{2+}$ in wastewater.

\section{Materials and methods}

\subsection{Materials}

$\beta$-CD (Shanghai Huishi Biochemical Reagent Co, Ltd.); CTS (deacetylation degree 80.0\%-95.0\%) (Sinopharm Chemical Reagent Co, Ltd.); citric acid, sodium dihydrogen phosphate (Beijing Chemical Plant); PEG 400, glutaraldehyde, base sodium chloride (Tianjin Guangfu Fine Chemical Research Institute); distilled water, hydrochloric acid, sodium hydroxide, ethanol, CM, dicresol orange, zinc powder, ammonium acetate (all analytically pure).

\subsection{Methods}

\subsubsection{Preparation of $\beta-C D P$}

CM (2 g), 1 g PEG-400, $0.25 \mathrm{~g} \mathrm{NaH}_{2} \mathrm{PO}_{4}$, and $10 \mathrm{~g} \beta$-CD were placed into $250 \mathrm{ml}$ a beaker. Distilled water was added and heated until all compounds were fully dissolved, and then the solution was placed in a 
constant-temperature air drying oven and removed after reaction completion. The resulting product was carefully ground in a porcelain emulsion, and then fully washed, suction filtration and dried with distilled water and anhydrous ethanol. The final dry product was carefully ground to obtain $\beta$-CDP ${ }^{[15-16]}$. The reaction time and temperature of the $\beta$-CDP were varied for single-factor tests to prepare $\beta$-CDP using the preparation conditions shown in Table 1.

Table 1. Single-factor experimental design of $\beta$-CDP preparation

\begin{tabular}{crrrrr}
\hline Factor & \multicolumn{5}{c}{ Level } \\
& 1 & 2 & 3 & 4 & 5 \\
\hline $\begin{array}{c}\text { Reaction time } \\
/ \mathrm{h}\end{array}$ & 3.5 & 4 & 4.5 & 5 & 5.5 \\
$\begin{array}{c}\text { Temperature } \\
/{ }^{\circ} \mathrm{C}\end{array}$ & 130 & 135 & 140 & 145 & 150 \\
\hline
\end{tabular}

\subsubsection{Preparation of CTS/ $\beta-C D P$ complex}

A solution of CTS in hydrochloric acid and $\beta$-CDP dissolved in distilled water were weighed, and the two solutions were poured into a beaker. While slowly mixing, 25\% glutaraldehyde solution was added to the beaker, and the mixture was stirred at a constant temperature. After reaction completion, the Precipitate was filtered, washed with ethanol, distilled water, and vacuum filtered. The filtrate was dried at $45{ }^{\circ} \mathrm{C}$ to a constant weight to obtain the CTS/ $\beta$-CDP complex as a brown powder ${ }^{[17]}$.

The CTS/ $\beta$-CDP complex was prepared using different reaction temperatures, $C$ TS-to- $\beta$-CDP mass ratios, reaction times, and amount of added glutaraldehyde. The preparation conditions are shown in Table 2 .

Table 2. Single-factor experimental design of CTS/ $\beta$-CDP complex preparation

\begin{tabular}{cccccc}
\hline & \multicolumn{5}{c}{ Level } \\
Factor & 1 & 2 & 3 & 4 & 5 \\
\hline $\begin{array}{c}\text { Temperature } \\
/{ }^{\circ} \mathrm{C}\end{array}$ & 60 & 70 & 80 & 90 & 100 \\
Mass ratio & $1: 10$ & $1: 5$ & $3: 10$ & $2: 5$ & $1: 2$ \\
$\begin{array}{c}\text { Reaction } \\
\text { time } / \text { min }\end{array}$ & 60 & 70 & 80 & 90 & 100 \\
$\begin{array}{c}\text { Glutaraldeh } \\
\text { yde addition } \\
\text { /mL }\end{array}$ & 1.0 & 1.5 & 2.0 & 2.5 & 3.0 \\
\hline
\end{tabular}

\subsubsection{Adsorption test and determination of optimal preparation conditions for CTS $/ \beta-C D P$ complex}

The $\mathrm{Zn}^{2+}$ content was determined by the reaction of zinc and xylenol orange to form a stable orange-red complex at a weakly acidic $\mathrm{pH}$ of 6 . The maximum absorption wavelength was $568 \mathrm{~nm}$, the zinc content ranged from 0-3.2 $\mu \mathrm{g} / \mathrm{mL}$, and the absorbance showed a good linear relationship ${ }^{[18]}$.

The $\mathrm{Zn}^{2+}$ standard curve was obtained by placing 1.0 , 3.0, 5.0, 7.0, and $9.0 \mathrm{~mL}$ of a $10 \mu \mathrm{g} / \mathrm{mL} \mathrm{Zn}^{2+}$ standard solution into a $25 \mathrm{~mL}$ volumetric flask. Then, $1.5 \mathrm{~mL}$ dimethylphenol orange and $3 \mathrm{~mL}$ acetic acid-ammonium acetate solution were successively added to adjust the absorbance, and then the $\mathrm{Zn}^{2+}$ standard curve was drawn.

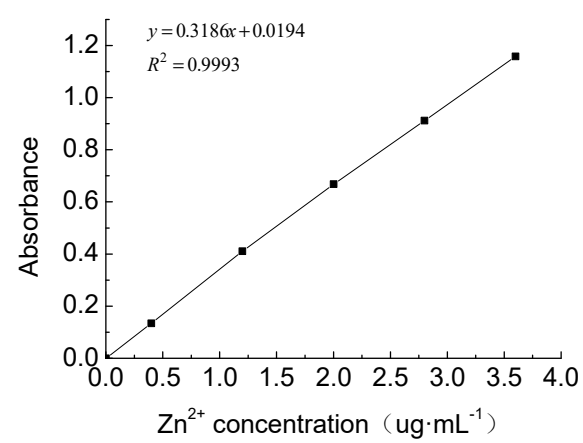

Fig. 1. $\mathrm{Zn}^{2+}$ concentration standard curve

The CTS/ $\beta$-CDP complex (0.2 g) obtained using different preparation conditions was added to $20 \mathrm{~mL}$ of 10 $\mu \mathrm{g} / \mathrm{mL} \mathrm{Zn}^{2+}$ standard solution, shaken at $100 \mathrm{r} / \mathrm{min}$ at a constant temperature for $2 \mathrm{~h}$, centrifuged, and then the supernatant was removed. The $\mathrm{Zn}^{2+}$ adsorption rate and adsorption amount were calculated by Equations 1 and 2:

$$
\begin{aligned}
& \boldsymbol{\eta}=\frac{\mathrm{C}_{0}-C_{t}}{\mathrm{C}_{0}} \times 100 \% \\
& \boldsymbol{R}=\frac{\left(\mathrm{C}_{0}-C_{t}\right) \times \mathrm{V}}{\mathrm{m}} \times 100 \%
\end{aligned}
$$

Where $C_{0}$ is the initial $\mathrm{Zn}^{2+}$ concentration, $C_{\mathrm{t}}$ is the concentration after constant-temperature oscillation for 2 $\mathrm{h}, \mathrm{V}$ is the volume of the $\mathrm{Zn}^{2+}$ solution, and $\mathrm{m}$ is the mass of the adsorbent. The $\mathrm{Zn}^{2+}$ adsorption capacity and adsorption rate of $\mathrm{CTS} / \beta-\mathrm{CDP}$ complexes were compared under different preparation conditions to determine the optimal preparation conditions of $\mathrm{CTS} / \beta-\mathrm{CDP}$ complexes.

\subsubsection{Comparative study on the $\mathrm{Zn}^{2+}$ adsorption capacity of CTS, $\beta-C D, \beta-C D P$, and $C T S / \beta-C D P$ Complex}

CTS, $\beta$-CD, and $\beta$-CDP $(0.2 \mathrm{~g})$ were weighed and added to $20 \mathrm{~mL}$ of $10 \mu \mathrm{g} / \mathrm{mL} \mathrm{Zn^{2+ }}$ standard solution, shaken at $100 \mathrm{r} / \mathrm{min}$ for $2 \mathrm{~h}$, centrifuged, and taken. The adsorption rate of the supernatant was determined and compared with the adsorption rate of the CTS/ $\beta$-CDP complex under its optimal adsorption conditions. 


\subsubsection{Fourier-transform infrared spectroscopy (FTIR) characterization}

CTS, $\beta$-CD, $\beta$-CDP, and CTS/ $\beta$-CDP complexes under their optimal adsorption conditions, and CTS/ $\beta$-CDP complex after $\mathrm{Zn}^{2+}$ adsorption were pressed into tablets with potassium bromide and analyzed using FTIR. The scanning range was $4000-500 \mathrm{~cm}^{-1}$. The infrared spectra of each substance were analyzed to determine the changes to surface groups before and after synthesis and adsorption.

\subsubsection{Scanning electron microscopy characterization}

(SEM)

CTS, $\beta-C D, \beta-C D P$, and $C T S / \beta-C D P$ complexes and $\mathrm{CTS} / \beta$-CDP complex after $\mathrm{Zn}^{2+}$ adsorption under optimal adsorption conditions were sprayed with gold on a copper sample holde to observe the morphology of the samples.

\section{Results and analysis}

\subsection{Effect of reaction conditions on the amount of $\beta$-CDP produced during the preparation of $\beta-C D P$}

\subsubsection{Effect of reaction time on the amount of $\beta-C D P$ produced}

Fig. 2 shows that when the reaction time was $4.5 \mathrm{~h}$, the amount of $\beta$-CDP produced reached $6.06 \mathrm{~g}$, and then decreased with longer reaction times. At longer reaction times, $\beta$-CDP partially decomposed, and therefore the optimum reaction time was set to $4.5 \mathrm{~h}$.

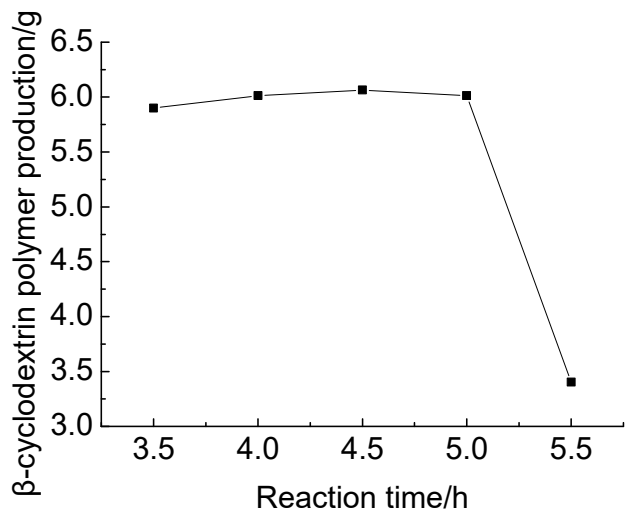

Fig. 2. Effect of reaction time on the amount of $\beta$-CDP produced

\subsubsection{Effect of reaction temperature on the amount of $\beta$-CDP produced}

Fig. 3 shows that when the reaction temperature was $145{ }^{\circ} \mathrm{C}$, the amount of $\beta$-CDP reached $6.58 \mathrm{~g}$, and then decreased when the reaction temperature was increased further. This occurred because at higher temperatures, the reaction rate was lower, which decreased the amount of product. Therefore, $145{ }^{\circ} \mathrm{C}$ was used as the optimum reaction temperature to prepare $\beta$-CDP.

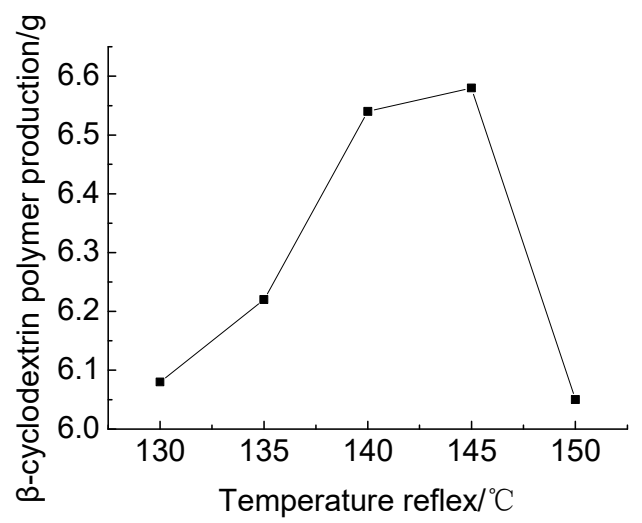

Fig. 3. Effect of reaction temperature on the amount of $\beta$-CDP produced

3.2 Effect of reaction conditions on the adsorption amount and adsorption rate of $\mathrm{Zn}^{2+}$ during the preparation of CTS/ $\beta$-CDP complex

\subsubsection{Effect of reaction temperature on $\mathrm{Zn}^{2+}$ adsorption and adsorption rate}

Fig. 4 shows that the adsorption amount and adsorption rate of $\mathrm{Zn}^{2+}$ increased with the reaction temperature. At a reaction temperature of $80^{\circ} \mathrm{C}$, the adsorption amount and adsorption rate reached their highest values of 34.93 $\mathrm{mg} \cdot \mathrm{g}^{-1}$ and $28.40 \%$, respectively. Then, when the temperature was increased further, the adsorption amount and adsorption rate gradually decreased. This occurred because the temperature was too high, and part of the complex decomposed, and the synergistic effect of CTS and $\beta$-cyclodextrin complex was reduced, decreasing the adsorption amount and adsorption rate. Thus, the optimum reaction temperature was $80^{\circ} \mathrm{C}$.

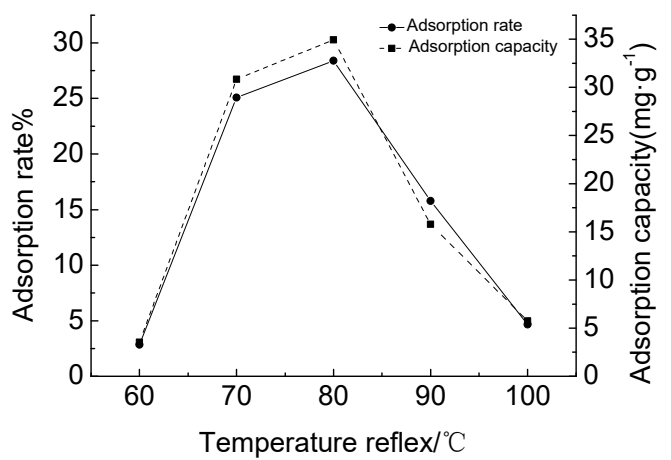

Fig. 4. Effect of reaction temperature on $\mathrm{Zn}^{2+}$ adsorption amount and adsorption rate

\subsubsection{Effect of mass ratio of CTS-to- $\beta$-CDP on $\mathrm{Zn}^{2+}$ adsorption amount and adsorption rate}

Fig. 5 shows that the adsorption amount and adsorption rate of $\mathrm{Zn}^{2+}$ continuously increased with the mass ratio 
of CTS-to- $\beta$-CDP. When the mass ratio reached 3:10, the adsorption amount and adsorption rate of $\mathrm{Zn}^{2+}$ reached their highest values of $97.70 \mathrm{mg} \cdot \mathrm{g}^{-1}$ and $78.92 \%$, respectively. However, they both decreased when the mass ratio of CTS-to- $\beta$-CDP was increased further because the number of hydroxyl groups on the composite structure increased. This caused the composite surface to become negatively charged, and the adsorption amount and adsorption rate of $\mathrm{Zn}^{2+}$ continuously increased. However, when a certain mass ratio was reached, the excess $\beta$-CDP cross-linked with itself or dissolved, so that the number of hydroxyl functional groups on the structure of the composite was continuously reduced. This decreased the adsorption rate and adsorption amount. Therefore, the optimal mass ratio of CTS-to- $\beta$-CDP was determined to be $3: 10$.

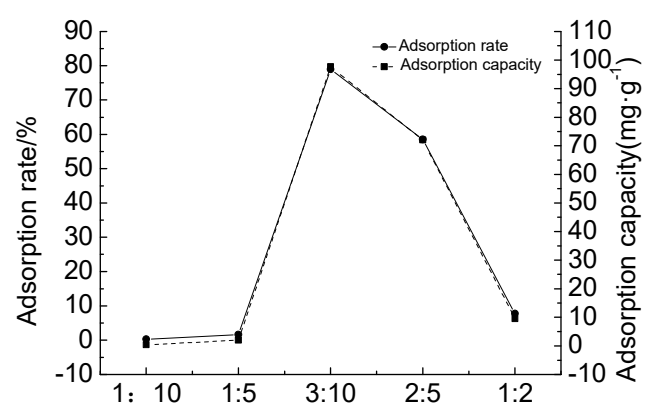

Chitosan, $\beta$-cyclodextrin polymer mass ratio

Fig. 5. Effect of mass ratio of CTS-to- $\beta-C D$ on the adsorption amount and adsorption rate of $\mathrm{Zn}^{2+}$

\subsubsection{Effect of reaction time on $\mathrm{Zn}^{2+}$ adsorption amount and adsorption rate}

It can be seen from Fig. 6 that the adsorption amount and the adsorption rate reached their highest values of 97.70 $\mathrm{mg} \cdot \mathrm{g}^{-1}$ and $78.92 \%$, respectively at a reaction time of 90 min. At longer reaction times, the adsorption amount and the adsorption rate decreased due to shell aggregation at $90 \mathrm{~min}$. The sugar completely reacted with the $\beta$-CDP, indicating that the optimum reaction time was $90 \mathrm{~min}$.

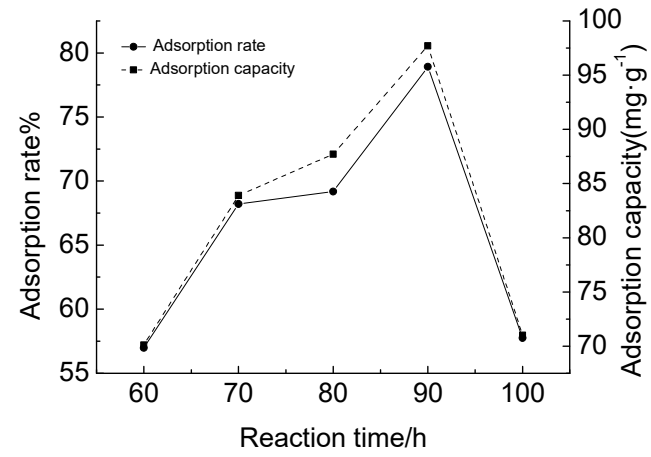

Fig. 6. Effect of reaction time on $\mathrm{Zn}^{2+}$ adsorption amount and adsorption rate

3.2.4 Effect of the glutaraldehyde amount on the adsorption amount and adsorption rate of $\mathrm{Zn}^{2+}$
Fig. 7 shows that the adsorption amount and the adsorption rate gradually increased as more glutaraldehyde was added, reaching a maximum of 97.70 $\mathrm{mg} \cdot \mathrm{g}^{-1}$ and $78.92 \%$ at $2 \mathrm{~mL}$. Then, the adsorption capacity and adsorption rate of $\mathrm{Zn}^{2+}$ decreased with further increases in the amount of glutaraldehyde. This is because excess glutaraldehyde occupied more CTS active sites and $\beta$-CDP, resulting in insufficient amounts of CTS and $\beta$-CDP to chelate with $\mathrm{Zn}^{2+}$. The optimum amount of glutaraldehyde in the reaction was therefore determined to be $2 \mathrm{~mL}$.

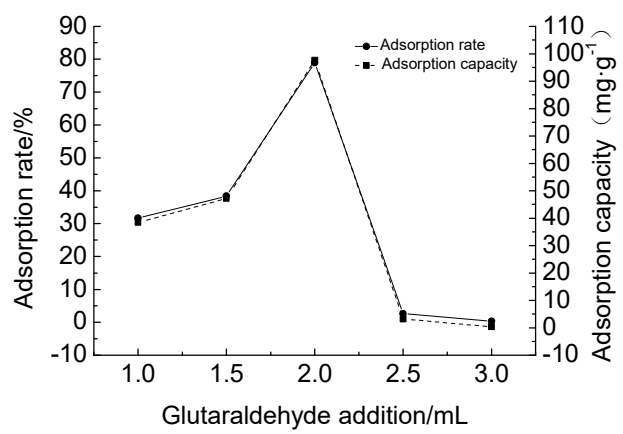

Fig.7. Effect of the amount of glutaraldehyde on the adsorption amount and adsorption rate of $\mathrm{Zn}^{2+}$

\subsection{Comparison of $\mathrm{Zn}^{2+}$ adsorption rates by $\beta-C D, \beta-C D P, C T S$, and CTS/ $\beta-C D P$ complex under optimal adsorption conditions}

Fig. 8 shows that under the same adsorption conditions, the adsorption rate and amount of CTS/ $\beta$-CDP complex on $\mathrm{Zn}^{2+}$ in solution were much higher than those of the other three substances. Thus, it can be concluded that CTS and $\beta-C D$ have synergistic effects during $\mathrm{Zn}^{2+}$ adsorption from waste water.

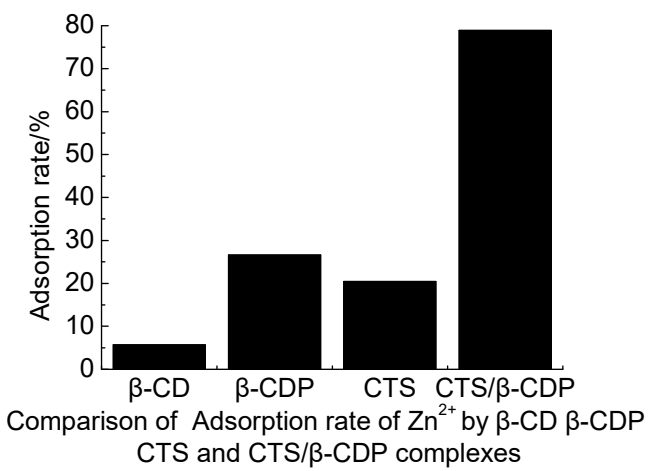

Fig. 8. Comparison of $\mathrm{Zn}^{2+}$ adsorption rates by $\beta-\mathrm{CD}, \beta-\mathrm{CDP}$, $\mathrm{CTS}$, and CTS/ $\beta$-CDP complex under optimal adsorption conditions

\subsection{FTIR analysis of $\beta-C D$ and $\beta-C D P$}

The FTIR spectra in Fig. 9 show that the characteristic absorption peaks of $\beta-C D$ and $\beta-C D P$ are roughly the same, with strong and broad peaks generated by the hydroxyl group stretching vibration near $3400 \mathrm{~cm}^{-1}$, and the characteristic absorption peaks of the $\alpha-(1,4)$ glycoside and $\alpha-(1,4)$ bonds of glucosamine at 937.65 
$\mathrm{cm}^{-1}$ and $860.63 \mathrm{~cm}^{-1}$, respectively. The infrared spectrum of $\beta$-CDP showed an ester peak absorption peak at $1708.33 \mathrm{~cm}^{-1}$. Thus, it was speculated that the carboxyl group of citric acid and the beta hydroxyl group reacted with one another to generate the ester bond of $\beta-C D$, confirming the successful synthesis of $\beta$-CDP.

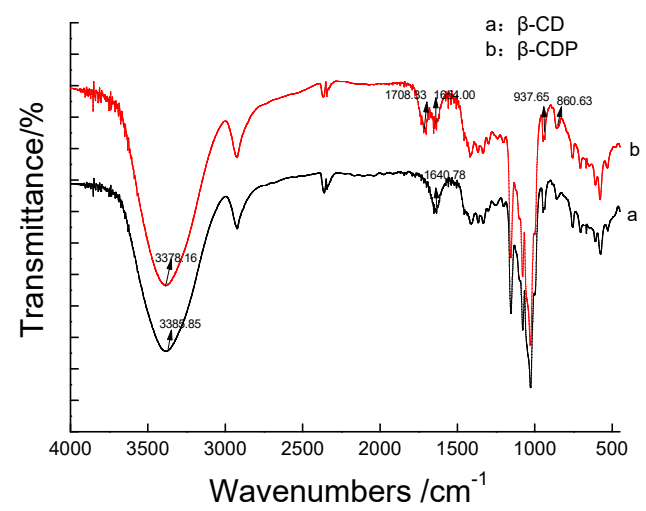

Fig. 9. FTIR spectra of $\beta-C D$ and $\beta-C D P$

\subsection{FTIR analysis of CTS, CTS/ $\beta$-CDP complex and $C T S / \beta-C D P$ complex after $\mathrm{Zn}^{2+}$ adsorption}

The FTIR spectra in Fig. 10 contain a peak at 3426.79 $\mathrm{cm}^{-1}$, which was attributed to the stretching vibration of the hydroxyl groups of CTS, and $895.72 \mathrm{~cm}^{-1}$ is a characteristic peak of the $\beta-(1,4)$ glycosidic bond of CTS. After the polymerization of $\beta-C D$, peaks appeared at $937.65 \mathrm{~cm}^{-1}$ and $860.63 \mathrm{~cm}^{-1}$, which corresponded to the $\alpha-(1,4)$ glycosidic linkages and $\alpha-(1,4)$ glucopyranose absorption peaks of $\beta$-CDP, respectively. The infrared spectra of the polysaccharide and $\beta$-CDP contain a characteristic peak at $895.43 \mathrm{~cm}^{-1}$ which was the $\beta-(1,4)$ glycosidic bond of CTS in the CTS/ $\beta$-CDP complex. The peaks at $947.57 \mathrm{~cm}^{-1}$ and $846.20 \mathrm{~cm}^{-1}$ appeared due to the $\alpha-(1,4)$ glycosidic linkages and the $\alpha-(1,4)$ glucopyranose absorption characteristic peaks of $\beta$-CDP, respectively. Since CTS and $\beta$-CDP have many of the same functional groups, when they were crosslinked, CTS masked the $\beta$-CDP peaks, from which it can be inferred that $\beta$-CDP was successfully polymerized. The chitin was cross-linked with CTS to obtain a CTS/ $\beta$-CDP complex.

By comparing the infrared spectra before and after $\mathrm{Zn}^{2+}$ adsorption, it can be seen that the absorption stretching peaks of the hydroxyl groups at 3404.90 $\mathrm{cm}^{-1}$ shifted to $3424.42 \mathrm{~cm}^{-1}$, and the amine deformation and bending vibration peaks at $1640.66 \mathrm{~cm}^{-1}$ and $1576.95 \mathrm{~cm}^{-1}$. After $\mathrm{Zn}^{2+}$ adsorption, these peaks increased and decreased to $1640.76 \mathrm{~cm}^{-1}$ and 1553.87 $\mathrm{cm}^{-1}$, respectively. The hydroxyl bending vibration peak near $1029.06 \mathrm{~cm}^{-1}$ disappeared after adsorbing $\mathrm{Zn}^{2+}$, and the absorption peaks at $947.57 \mathrm{~cm}^{-1}$ and $846.20 \mathrm{~cm}^{-1}$ also disappeared. It can be inferred that the $\beta$-CDP and $\mathrm{Zn}^{2+}$ of the complex also reacted, confirming that the active groups on the CTS/ $\beta$-CDP complex were involved in adsorption based on these peak changes. Thus, the complex was synthesized and reacted with $\mathrm{Zn}^{2+}$.

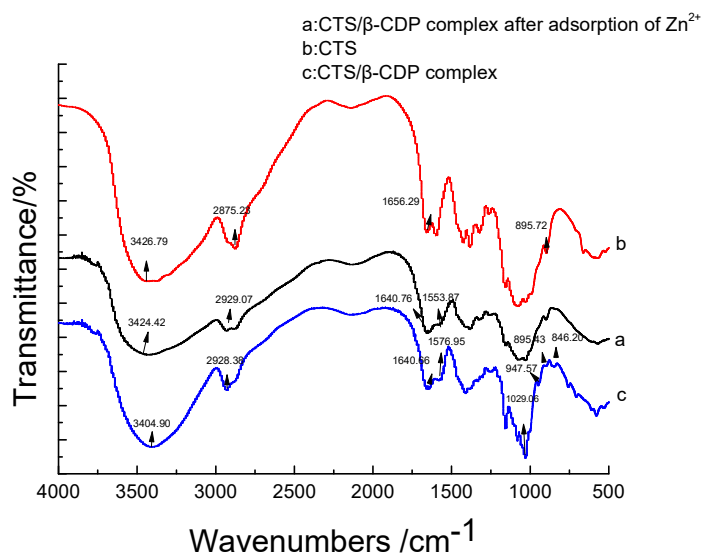

Fig.10. FTIR spectra of CTS, CTS $/ \beta$-CDP complex, and $\mathrm{CTS} / \beta-\mathrm{CDP}$ complex after $\mathrm{Zn}^{2+}$ adsorption

\subsection{SEM analysis of $\beta-C D, \beta-C D P, \quad C T S$, CTS/ $\beta$-CDP complex, and CTS/ $\beta$-CDP complex after $\mathrm{Zn}^{2+}$ adsorption}

As seen in Fig. 11, the surface of the $\beta-C D$ was smooth and had a plate-like shape with many particles, while the modified $\beta$-CDP showed agglomeration and contained pores on its surface. The CTS had a flaky structure with a smooth surface and no wrinkles. The CTS/ $\beta-C D P$ complex had a solid particle structure, with a tight and rough surface, with a higher specific surface area than CTS, and an increased number of adsorption sites. After $\mathrm{Zn}^{2+}$ adsorption, the surface of the CTS/ $\beta$-CDP complex became rougher, and it is speculated that many $\mathrm{Zn}^{2+}$ ions were adsorbed on its surface.
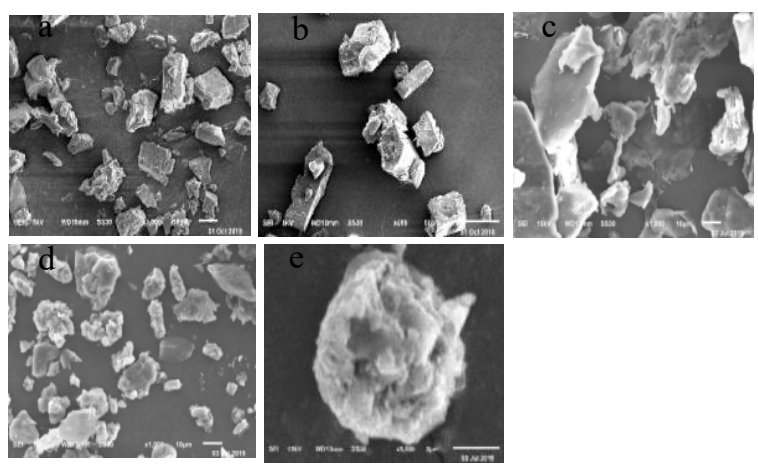

a: $\beta-C D(50 \mu \mathrm{m})$; b: $\beta-C D P(50 \mu \mathrm{m})$; c: CTS $(10 \mu \mathrm{m})$; d: CTS/ $\beta$-CDP complex $(10 \mu \mathrm{m})$; e: CTS/ $\beta$-CDP complex after $\mathrm{Zn}^{2+}$ adsorption $(5 \mu \mathrm{m})$

Fig. 11. SEM analysis of $\beta-C D, \beta-C D P, C T S, C T S / \beta-C D P$ complex, and CTS/ $\beta$-CDP complex after $\mathrm{Zn}^{2+}$ adsorption

\section{Conclusion}

In this study, the optimum conditions for the adsorption of $\mathrm{Zn}^{2+}$ from waste water using a synthesized CTS/ $\beta$-CDP complex were determined to be: a temperature of $80^{\circ} \mathrm{C}$, a mass ratio of CTS-to- $\beta$-CDP of $3: 10$, a reaction time of $90 \mathrm{~min}$, and $2 \mathrm{~mL}$ glutaraldehyde. 
The adsorption amount and adsorption rate of $\mathrm{Zn}^{2+}$ using the obtained complex reached a maximum of 97.70 $\mathrm{mg} \cdot \mathrm{g}^{-1}$ and $78.92 \%$. In addition, the adsorption of $\mathrm{Zn}^{2+}$ in waste water using the $\mathrm{CTS} / \beta$-CDP complex was much stronger than that of CTS, $\beta-\mathrm{CD}$, or $\beta$-CDP under the same adsorption conditions.

The FTIR spectral analysis showed that an ester absorption peak at $1708.33 \mathrm{~cm}^{-1}$ formed from the reaction of the carboxyl group of citric acid with the hydroxyl group on $\beta-C D$, indicating the successful synthesis of $\beta$-CDP. Peaks of the $\beta-(1,4)$ glycosidic bond of CTS appeared in the infrared spectrum of CTS/ $\beta-C D P$ at $895.43 \mathrm{~cm}^{-1}$ and $947.57 \mathrm{~cm}^{-1}$, while $846.20 \mathrm{~cm}^{-1}$ was from the $\beta$-CDP $\alpha-(1,4)$ glycosidic bond and $\alpha-(1,4)$ glucopyranose absorption characteristic peak, indicating the successful synthesis of the CTS/ $\beta$-CDP complex. The FTIR spectra before and after $\mathrm{Zn}^{2+}$ adsorption were different, and some characteristic peaks disappeared or shifted due to the adsorption of $\mathrm{Zn}^{2+}$. SEM analysis showed that $\beta$-CDP was tighter than $\beta-C D$ itself, and the surface of the CTS/ $\beta$-CDP complex was rougher than CTS itself. The adsorption sites increased which increased the adsorption rate. Additionally, the CTS/ $\beta$-CDP complex became rougher after adsorption due to the accumulation of many $\mathrm{Zn}^{2+}$ ions on its surface.

CTS and $\beta-C D$ are natural products, and a CTS/ $\beta$-CDP complex was prepared to adsorb $\mathrm{Zn}^{2+}$ by utilizing the metal ion adsorption capacity of CTS and the cavity structure of $\beta-\mathrm{CD}$. When used to adsorb $\mathrm{Zn}^{2+}$ from waste water, the CTS/ $\beta$-CDP complex was slightly more efficient than either CTS or $\beta-\mathrm{CD}$ individually. Additionally, this adsorption process is simple and low-cost, and cannot cause secondary pollution to the environment.

\section{Fund Project}

Research and Cultivation Project of Changchun University (ZKQ201911)

Changchun University Masters Academic innovation project (Scavenging effect of chitosan/ $\beta$-cyclodextrin composite membrane on chloride ion)

\section{References}

1. Y.H.Xie, Q.Zhao, J.S.Yuan. Research on removing zinc ions from retrofic clinoptilolite in water. J Salt Chem Ind,42(12):21-25(2013)

2. L.Chen,X.Qiang,D.B.Cheng.Research on modification and application of sepiolite in lead and zinc contained wastewater treatment. PLATING \& FINISHING,37(01):19-26(2015)

3. J.Zhou. Adsorption of lead, zinc, and cadmium ions from aqueous solution by teawaste adsorbents. Hunan university(2013)

4. Y.L.Shen, et al. New technology for wastewater biological treatment. China Environmental Science Press, 88-90(1999)

5. T.Wang,L.Chen, T.Shen, D.Wu. Preparation and properties of a novel thermo sensitive drogel based on chitosan/hydroaypropyl methylcellulose/glycerol. INT J BIOL MACROMOL, 93(A): 775-782(2016)

6. B.Li,L.Yang,C.Wang,Q.Zhang,Q.Li ,Y.Li, R.Xiao. Adsorption of $\mathrm{Cd}$ (II) from aqueous solutions by rape straw biochar derived from different modification processes. CHEMOSPHERE, 175: 332-340(2017)

7. W.Liang, M.Li , Z.Zhang, et al. Decontamination of $\mathrm{Hg}$ (II) from aqueous solution using polyamine-co-thiourea marched chitosan gel derivatives .INT J BIOL MACROMOL(2018)

8. U.G.Beker, F.S.Guner,et al. Heavy metal removal by ion exchanger based on hydroxyethyl cellulose. J APPL POLYM SCI, 74(14):3501-3506(1999)

9. X.Tian,J.Zhang,X.Jiang. Modification of $\beta$-cyclodextrin for rapidly decolorizing the dye-containing wastewaters by flocculation .J APPL POLYM SCI,131(6):8(2014)

10. K.Moue,K.Yoshizuka,K.Ohto.Adsorptive separation of some metal ions by complexing agent types of chemically modified chitosan. ANAL CHIM ACTA, 388(1-2):209-218(1999)

11. Y.Vijaya, S.R.Popuri, V.M.Boddu, et al. Modified chitosan and calcium alginate biopolymer sorbents for removal of nickel (II) through adsorption. CARBOHYD POLYM, 72(2): 261-271(2008)

12. E.Repo , J.K.Warchol, T.A.Kurniawan, et al. Adsorption of Co (II) and Ni (II) by EDTA-and/or DTPA-modified chitosan: kinetic and equilibrium modeling.CHEM ENG J, 161(1-2): 73-82(2010)

13. Y.N.Hu, Synthesis and properties of cyclodextrin grafted chitosan derivatives . Shenyang Pharmaceutical University (2009)

14. M.E.Skold,G.D.Thyne,J.W.Drexler,etal. Enhanced Solubilization of a Metal-Organic Contaminant Mixture (Pb, Sr, Zn, and Perchloroethylene) by Cyclodextrin. ENVIRON SCI TECHNOL, 42(23):8930-8934(2008)

15. D.Yu, L.I.Wu, J.Yang, et al. Preparation of $\beta-C D / C S$ membrane and its application to wastewater treatment . Dye Finis, 39(2):15-18+45(2013)

16. N.Wu. The preparation and research on cupric wastewater of $C S / \beta-C D P / T i \quad O_{2}$ membrance. Nanjing University of Aeronautics and Astronautics(2015)

17. H.X.Gu. Adsorption of chromium and cadmium by chitosan- $\beta$-cycl odextrin in water. University of Shanghai for Science and Technology,(2014)

18. C.X.Gao, M.Zhu.Determination of Zinc in Cyclic Wastewater by Spectrophotometry.Shanghai E. S.(04):24-25(1994) 